OPEN ACCESS

Edited by:

Zhiming Pan,

Yangzhou University, China

Reviewed by:

Guoqiang Zhu,

Yangzhou University, China

V. K. Viswanathan,

University of Arizona, United States

*Correspondence:

Rachel A. Cheng

ram524@cornell.edu

Specialty section: This article was submitted to

Bacteria and Host,

a section of the journal

Frontiers in Cellular and

Infection Microbiology

Received: 10 November 2020

Accepted: 21 December 2020

Published: 03 February 2021

Citation:

Cheng RA and Wiedmann M (2021)

Recent Advances in

Our Understanding of the

Diversity and Roles of

Chaperone-Usher Fimbriae in

Facilitating Salmonella Host

and Tissue Tropism.

Front. Cell. Infect. Microbiol. 10:628043.

doi: 10.3389/fcimb.2020.628043

\section{Recent Advances in Our}

Understanding of the Diversity and Roles of Chaperone-Usher Fimbriae in Facilitating Salmonella Host and Tissue Tropism

\author{
Rachel A. Cheng* and Martin Wiedmann \\ Department of Food Science, Cornell University, Ithaca, NY, United States
}

Salmonella enterica is one of the most diverse and successful pathogens, representing a species with $>2,600$ serovars with a variety of adaptations that enable colonization and infection of a wide range of hosts. Fimbriae, thin hair-like projections that cover the surface of Salmonella, are thought to be the primary organelles that mediate Salmonella's interaction with, and adherence to, the host intestinal epithelium, representing an important step in the infection process. The recent expansion in genome sequencing efforts has enabled the discovery of novel fimbriae, thereby providing new perspectives on fimbrial diversity and distribution among a broad number of serovars. In this review, we provide an updated overview of the evolutionary events that shaped the Salmonella chaperone-usher fimbriome in light of recent phylogenetic studies describing the population structure of Salmonella enterica. Furthermore, we discuss the complexities of the chaperone-usher fimbriae-mediated host-pathogen interactions and the apparent redundant roles of chaperone-usher fimbriae in host and tissue tropism.

Keywords: Salmonella, fimbriae, chaperone-usher, host-pathogen interaction, adhesin Salmonella

\section{INTRODUCTION}

The World Health Organization reported that Salmonella infections contributed the greatest burden of foodborne disease of any diarrheal disease agent, resulting in an estimated 8.6 million [95\% confidence interval (CI) 3.9-17.5 million] disability-adjusted life years (DALYs) (Havelaar et al., 2015). Salmonella includes just two different species (S. bongori and S. enterica), but at least 2,659 known serovars (Issenhuth-Jeanjean et al., 2014). Among the six recognized subspecies of S. enterica (Brenner et al., 2000), the majority of serovars associated with human clinical disease belong to $S$. enterica subsp. enterica (Centers for Disease Control and Prevention, 2016). Serovars are often divided into typhoidal (serovar Typhi), paratyphoidal (serovars Paratyphi A, B, and C, and Sendai/ Miami) and nontyphoidal serovars (e.g., serovars Typhimurium and Enteritidis), to reflect the diseases that they cause, with infection with typhoidal and paratyphoidal serovars resulting in an invasive, extraintestinal infection, and infection with nontyphoidal serovars resulting in a primarily 
gastrointestinal illness that is often self-limiting (Crump et al., 2015). An ongoing challenge in the effort to reduce the morbidity and mortality associated with human salmonellosis, is that many Salmonella serovars are known to colonize and infect a wide range of hosts, although some serovars are host-restricted (e.g., Typhi in humans) or host-adapted (e.g., Dublin in cows). Furthermore, potential reservoirs of many nontyphoidal serovars that are commonly associated with human clinical illness, remain largely unknown.

Reflective of its overall success at colonizing a large range of hosts to enhance its distribution throughout many environments, Salmonella uses a variety of strategies to survive passage through the host gastrointestinal tract, enabling its transient or long-term presence, and facilitating its spread to additional host populations. Fimbriae (also known as pili) are the thin, hair-like appendages that mediate bacterial adherence to a surface, such as the intestinal epithelium (Nuccio and Bäumler, 2007), although non-fimbrial adhesins have also been described (Wagner and Hensel, 2011). Given the importance of fimbriae in mediating host interactions for multiple pathogens (Kaper et al., 2004; Nobbs et al., 2009; Paczosa and Mecsas, 2016), special attention has been given to characterizing the role that different Salmonella fimbriae play in mediating host and tissue tropism, as well as their potential use in source attribution predictions (Zhang et al., 2019).

The recent increases in the number of Salmonella whole genome sequences (WGS) available has afforded the discovery and characterization of fimbriae encoded by a variety of Salmonella (Nuccio and Bäumler, 2007; Worley et al., 2018), as most fimbriae are poorly expressed under standard culturing conditions (Humphries et al., 2003; Thanassi et al., 2007; Hansmeier et al., 2017). In this review, we discuss recent advances in our understanding of the content and evolution of the Salmonella chaperone-usher fimbriome, and evidence supporting the complexity of fimbriae-mediated interactions in the context of tissue and host tropism.

\section{A COLLECTOR'S ITEM: SALMONELLA ENCODES A DIVERSE NUMBER OF CHAPERONE-USHER FIMBRIAE}

Since the discovery of fimbriae in Escherichia coli in 1955, at least 39 different fimbriae have been described in Salmonella (Yue et al., 2012; Desai et al., 2013; Aviv et al., 2017; Cheng et al., 2019; Rehman et al., 2019). Fimbriae belong to four general categories depending on their morphology, function, or assembly pathway: (i) F pili, (ii) type IV fimbriae, (iii) curli, and (iv) chaperoneusher pathway fimbriae (reviewed in [Nuccio and Bäumler, 2007)]. Fimbriae of the chaperone-usher pathway represent the majority of fimbriae encoded by Salmonella (Nuccio and Bäumler, 2007; Rehman et al., 2019), and are therefore the focus of our discussion. Chaperone-usher pathway fimbriae, so named because these fimbriae utilize one or more chaperones to transfer folded fimbrial subunits to the usher protein (an integral outer membrane protein) for translocation across the outer membrane (Figure 1A), are categorized phylogenetically based on sequence conservation of the usher protein (Nuccio and Bäumler, 2007; Thanassi et al., 2007). The number of genes within a given chaperone-usher fimbrial gene cluster varies, but all include genes encoding (i) an usher protein, (ii) at least one chaperone, and (iii) a major fimbrial structural subunit; many fimbriae also include a minor fimbrial structural subunit known as a tip adhesin (Nuccio and Bäumler, 2007).

In 2007, Nuccio and Bäumler proposed the current classification scheme for chaperone-usher pathway fimbriae, which groups chaperone-usher fimbriae into three general categories based on the phylogenetic relatedness of the usher protein: (i) alternate, or $\alpha$-fimbriae, (ii) classical, or $\beta-, \gamma_{-}, \kappa-, \pi$ fimbriae; (iii) and archaic, or $\sigma$-fimbriae (Nuccio and Bäumler, 2007). Members of the $\gamma$-fimbriae are further sub-divided into classes $\gamma_{1}$ through $\gamma_{4}$ (Nuccio and Bäumler, 2007), although $\gamma_{2}$ fimbriae have not been identified in Salmonella (Yue et al., 2012; Rehman et al., 2019). Among the 36 known chaperone-usher fimbriae produced by Salmonella, the alternative and archaic fimbriae include just one member each (Yue et al., 2012), and the remaining 34 represent members of the classical fimbriae with 23 belonging to the $\gamma$-fimbriae (Figure 1B) (Desai et al., 2013; Rehman et al., 2019). Additional novel fimbriae have since been proposed but the fimbrial gene cluster family for those was not reported (Desai et al., 2013; Aviv et al., 2017).

\section{EVOLUTION OF THE SALMONELLA CHAPERONE-USHER FIMBRIOME: A STORY OF GAIN AND LOSS}

Recent advances in our understanding of the Salmonella chaperone-usher fimbriome have been catalyzed by the appreciable increase in the number of WGS data available. A synthesis of several studies (Fookes et al., 2011; Yue et al., 2012; Desai et al., 2013; Worley et al., 2018) examining the presence of fimbrial gene clusters among serovars belonging to the major lineages of Salmonella (Worley et al., 2018) suggests a complex history of multiple gain and loss events (Figure 1B).

\section{The Ancestral Chaperone-Usher Fimbriome: Fimbrial Gene Clusters Acquired Before the Divergence of Salmonella and Escherichia}

At least seven Salmonella chaperone-usher fimbrial gene clusters are orthologous to fimbriae characterized in Escherichia (Nuccio and Bäumler, 2007), suggesting that these fimbrial gene clusters were most likely acquired prior to the divergence of these two genera approx. 140 million years ago (Yue et al., 2012; Desai et al., 2013). The Bcf fimbrial gene cluster [orthologue of Ycb in E. coli (De Masi et al., 2017)], originally named for its role in colonization of bovine hosts (Tsolis et al., 1999), is present in $S$. bongori and S. enterica, although this fimbrial gene cluster has been lost in subspecies houtenae (Desai et al., 2013). The conservation of a Fim (also called Type I fimbriae) orthologous fimbrial gene cluster [called Sfm (for Salmonella like fimbriae) 


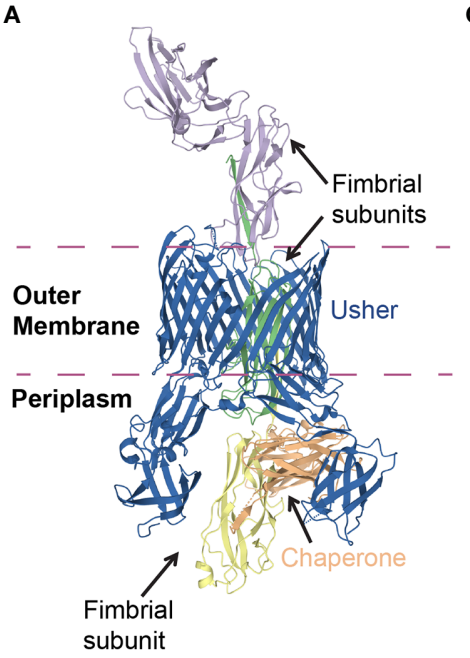

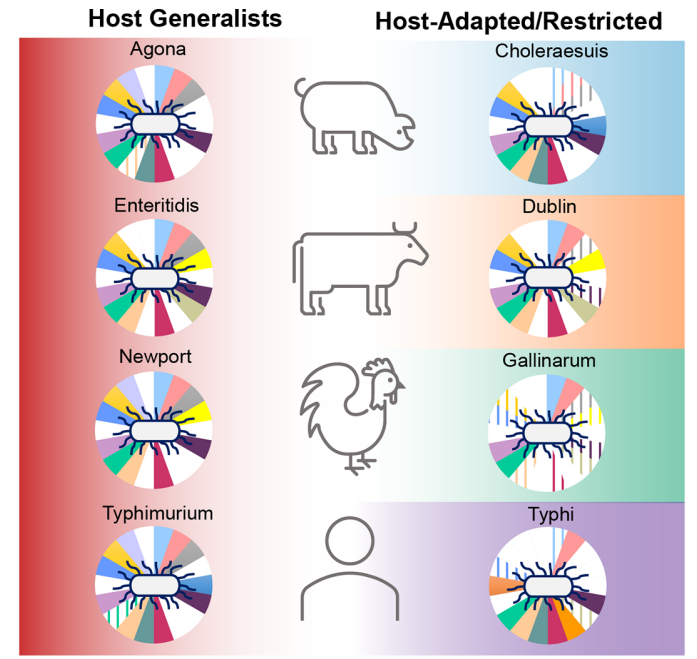

B

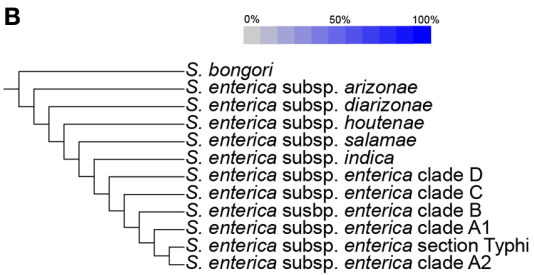

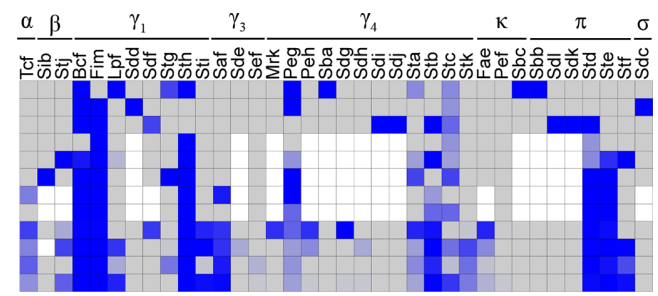

FIGURE 1 | An overview of the Salmonella chaperone-usher fimbriome. (A) Structure of a chaperone-usher protein complex. The structure for Fim fimbria is shown as an example (PDB accession: 4J3O) (Geibel et al., 2013) to demonstrate the chaperone-usher biogenesis pathway. The membrane embedded usher (blue; FimD) accepts folded fimbrial subunits (FimF, FimG, and FimH) from the chaperone (orange; FimC) and translocates them across the outer membrane for elongation of the fimbria on the cell surface. (B) Overview of the distribution of known chaperone-usher fimbriae in Salmonella. Blue shading indicates the proportion of isolates in a given group that encoded the fimbrial gene cluster. White squares represent fimbrial gene clusters that were not included in analyses for a given Salmonella clade. The number of isolates included for each comparison varied: S. bongori (1-3 isolates), S. enterica subsp. arizonae and diarizonae (1-7 isolates), S. enterica subsp. houtenae (2-7 isolates), S. enterica subsp. salamae (2-9 isolates), S. enterica subsp. indica (1-3 isolates), and S. enterica subsp. enterica clades D (8 isolates), C (4 isolates), B (5-138 isolates), A1 (6-67 isolates), section Typhi (3-24 isolates), and A2 (10-186 isolates). For Tcf, Agf, Bcf, Fim, Lpf, Stg, Sth, Sti, Saf, Sef, Peg, Sta, Stb, Stc, Stk, Pef, Std, Ste, and Stf fimbriae, proportions of isolates in S. enterica subsp. enterica reflect only isolates from (Worley et al., 2018); for subsp. arizonae, diarizonae, houtenae, salamae, and indica datasets from multiple studies were compiled (Yue et al., 2012; Desai et al., 2013; Worley et al., 2018) as low numbers of isolates for these subspecies were reported. For S. bongori, data were compiled from (Fookes et al., 2011; Yue et al., 2012; Desai et al., 2013). Finally, for fimbriae Sdf, Sdg, Stj, Sdd/Smf, Sde, Mrk, Peh, Sba, Sdh, Sdi, Sdj, Fae, Sbc, Sbb, Sdk, Sdi, and Sdc, data were compiled from (Fookes et al., 2011; Yue et al., 2012; Desai et al., 2013). Fimbriae were considered as "present" if at least half of the genes in the operon were detected; pseudogenes were not considered in this analysis. In the Desai et al. (2013) dataset, Fae fimbria was referred to as "Skf" and Sdc fimbria was referred to as "Sas". (C) A closer look at the distribution of chaperone-usher fimbriae among host-restricted (Gallinarum and Typhi), host-adapted (Choleraesuis and Dublin), and broad host range (Agona, Enteritidis, Newport, and Typhimurium) serovars. Background colors show associations with hosts (host generalists are shown in red to signify that they can infect all hosts shown, hostadapted/restricted serovars are aligned with the host that they are adapted/restricted to) (Hoelzer et al., 2011). Fimbriae are represented by the pie charts behind each Salmonella, with each slice of the chart representing an individual fimbria (clockwise from top light blue slice: Bcf, Fim, Lpf, Peg, Pef, Saf, Sef, Sta, Stb, Stc, Std, Ste, Stf, Stg, Sth, Sti, Stj, and Stk); slices appear colored in if the fimbria is present (i.e. white signifies absence of the fimbria) or have lines if (i) the fimbria is predicted to include hypothetically disrupted coding sequences for the gene encoding the usher protein or (ii) if more than half of the genes in the fimbrial gene cluster are predicted to be missing or are hypothetically disrupted coding sequences (Nuccio and Bäumler, 2014). Figure is based on data from (Nuccio and Bäumler, 2014) and reflects data for S. Agona SL483, S. Enteritidis P125109, S. Newport SL476, S. Typhi CT18, S. Typhimurium LT2, S. Gallinarum 287/91, S. Choleraesuis SC-B67, and S. Dublin CT_02021853.

among many lineages in E. coli (Wurpel et al., 2013)] supports that this fimbrial gene cluster was acquired prior to the divergence of Salmonella and Escherichia (Townsend et al., 2001), although this fimbrial gene cluster has since been lost in S. bongori (Fookes et al., 2011; Yue et al., 2012; Desai et al., 2013). Long polar fimbria [Lpf (Bäumler and Heffron, 1995)] shows a distinct evolutionary pattern characterized by an apparent acquisition from E. coli [also called Lpf in E. coli (Torres et al.,
2002)] and conservation among S. bongori and some isolates of subspecies salamae and enterica (Worley et al., 2018); however some models based on a smaller set of isolates suggest the independent acquisition of the Lpf gene cluster by S. bongori and S. enterica subsp. enterica clade A serovars (Desai et al., 2013). The Peg fimbrial gene cluster [named for its original discovery in S. enterica subsp. enterica serovars Paratyphi A, Enteritidis, and Gallinarum (Thomson et al., 2008)] is detected 
broadly across both species and most $S$. enterica subspecies except for diarizonae and houtenae. S. bongori is predicted to have acquired the Sba fimbrial gene cluster from a most recent common ancestor (MRCA) shared with E. coli, although Sba has since been lost in S. enterica (Fookes et al., 2011; Yue et al., 2012). Finally, Sta [orthologue of E. coli Yad (Townsend et al., 2001; Wurpel et al., 2013)] and Stc [orthologue of E. coli Yeh (Townsend et al., 2001; Wurpel et al., 2013)] fimbrial gene clusters are found in S. bongori and S. enterica but show different distributions among the $S$. enterica subspecies (Figure 1B), suggesting different patterns of loss for these two fimbrial gene clusters (Yue et al., 2012; Desai et al., 2013).

\section{The Modern Chaperone-Usher Fimbriome: Fimbrial Gene Clusters (Likely) Acquired After Divergence From Escherichia}

Following Salmonella's divergence from Escherichia, the remaining chaperone-usher fimbrial gene clusters are hypothesized to have been acquired via horizontal gene transfer. While the exact mechanisms responsible for acquisition of the majority of Salmonella fimbrial gene clusters remain elusive, the presence of orthologous clusters in other Gammaproteobacteria (Nuccio and Bäumler, 2007; Desai et al., 2013; Stubenrauch et al., 2017), and the observation that multiple chaperone-usher fimbrial genes clusters are carried on plasmids [e.g., Pef and א-fimbriae (Bäumler et al., 1996a; Nuccio and Bäumler, 2007; Aviv et al., 2017)] support their horizontal acquisition from other genera.

Although originally characterized in S. enterica subsp. enterica serovars Typhi [Stb, Std, Ste, Stf, Stg, and Sth (Townsend et al., 2001)] and Typhimurium [Stj (Mcclelland et al., 2001)], these fimbriae are distributed widely across multiple different Salmonella lineages (Figure 1B). The Stb and Std fimbrial gene clusters were likely acquired by subspecies diarizonae (Yue et al., 2012) and maintained in the majority of subspecies enterica isolates. The evolutionary history of Sdf, Ste, Stf, Stg, and Stj fimbrial gene clusters likely involved multiple acquisition events or a combination of acquisition and loss/ retention by some subspecies (Yue et al., 2012; Desai et al., 2013). Last, the Sth fimbrial gene cluster is present in S. bongori and in most S. enterica subspecies (missing in subspecies arizonae and diarizonae); this fimbrial gene cluster was most likely acquired by S. bongori following its divergence from Escherichia (Desai et al., 2013) as this genomic region is missing from E. coli (Townsend et al., 2001) and no known E. coli fimbrial gene clusters with similar sequence homology have been identified (Nuccio and Bäumler, 2007).

The remaining Salmonella chaperone-usher fimbriae include those that have only been characterized in one species or subspecies. Multiple fimbrial gene clusters are found exclusively in non-subsp. enterica isolates: (i) Sbb and Sbc in $S$. bongori (Fookes et al., 2011; Yue et al., 2012), (ii) Sdd and Sdc, the only known $\sigma$-fimbria, in S. enterica subsp. arizonae, (iii) Sdi, Sdj, Sdl, and Sdk in S. enterica subsp. diarizonae (Yue et al., 2012), and (iv) Sib, a novel $\beta$-fimbria, in S. enterica subsp. indica (Desai et al., 2013). S. enterica subsp. enterica encodes 12 fimbriae that are not found in S. bongori or other S. enterica subspecies (Figure 1B). Tcf [for Typhi colonization factor (Folkesson et al., 1999)] and Saf are present in isolates representing all currently known subsp. enterica clades except for clade C. Sti, Peh, Sdg, and Sdh fimbrial gene clusters are found in multiple subsp. enterica clades, but are largely missing from section Typhi isolates (Sti is present in one serovar, Kintambo, in section Typhi), suggesting the selective loss of these fimbriae in section Typhi isolates. Conversely, Stk and Fae fimbrial gene clusters are found in some isolates representing subsp. enterica clades B, A1, Typhi, and A2. The plasmidencoded Pef fimbrial gene cluster was only detected in a handful of serovars including Nottingham (clade B) and four serovars in clade A2 that had previously been shown to carry the Salmonella virulence plasmid (i.e., serovars Choleraesuis, Enteritidis, Bovismorbificans, and Typhimurium) (Cheng et al., 2019). The Sef [Salmonella Enteritidis fimbriae (Clouthier et al., 1993)] fimbrial gene cluster is found in multiple clade A2 serovars as well as in some section Typhi isolates (Yue et al., 2012). Finally, the Sde and Mrk [Mannose resistant Klebsiellalike fimbriae (Wilksch et al., 2011)] fimbrial gene clusters have only been reported in serovars Tennessee (clade A1) and Montevideo (clade B), respectively (Yue et al., 2012).

With a more complete picture of the Salmonella chaperoneusher fimbriome, several key themes arise. First, many chaperone-usher fimbrial gene clusters are detected broadly across both $S$. bongori and S. enterica, suggesting the early acquisition of these chaperone-usher fimbrial gene clusters. Second, very few chaperone-usher fimbrial gene clusters are serovar-specific. While this may be a reflection of the specific bioinformatic approaches used (i.e., identity cut-off used) or the isolates in the comparison, it suggests that while fimbriae may contribute to host/niche adaptation, they are likely only a part of the process as many other factors also play a role (e.g., changes in metabolic pathways (Nuccio and Bäumler, 2014), immunological naivety of the host (Bäumler et al., 2000), etc.). Last, the currently available data highlight multiple "rare" fimbrial gene clusters, where future characterizations including a broader range of isolates will be necessary to extend our knowledge of the evolutionary events associated with these fimbriae.

\section{MAKING AN ENTRANCE-EXAMINING A ROLE FOR CHAPERON-USHER FIMBRIAE IN MEDIATING INTERACTIONS WITHIN AND BETWEEN HOSTS}

As reviewed previously (Rivera-Chavez and Bäumler, 2015), Salmonella is ill-suited to compete with the resident anaerobic microbiota within the lumen of the gut (Tsolis and Bäumler, 2020). Therefore, Salmonella uses different strategies upon entering the gut to either escape to a slightly less competitive environment or modify the environment to one that favors its expansion (Tsolis and Bäumler, 2020). Fimbriae are proposed to play a key role in mediating initial interactions with host cells 
(Fàbrega and Vila, 2013). The majority of the host cell surface receptors that are recognized and bound by fimbriae remain elusive, although several surface glycans have been identified as facilitating adhesion (Table 1): Std ( $\pi$-fimbriae) binds to terminal $\alpha-1,2$ fucose (Chessa et al., 2009; Suwandi et al., 2019), Fim ( $\gamma_{1}$-fimbriae) binds to mannose (Duguid et al., 1966), and Pef ( $\kappa$-fimbriae) binds Gal $\beta 1-4$ (Fuc $\alpha 1-3)$ GlcNAc [also called the $\mathrm{Le}^{\mathrm{x}}$ histo-blood group antigen (Chessa et al., 2008)]. These observations suggest that fimbriae may mediate preferential binding to different glycans, thereby facilitating Salmonella's ability to bind to different host cell surfaces either within the same host (tissue tropism) or in different hosts (host tropism).

\section{A Role for Chaperone-Usher Fimbriae in Tissue Tropism?}

Salmonella strains encode an average of 5-14 different fimbrial gene clusters (McClelland et al., 2001; Townsend et al., 2001; Yue et al., 2012; Worley et al., 2018) with multiple fimbriae being coexpressed in vivo (Humphries et al., 2003; Laniewski et al., 2017). During an infection, nontyphoidal Salmonella (e.g., S. Typhimurium) capitalize on a pro-inflammatory response to generate host-derived nitrate released by immune cells (RiveraChavez and Bäumler, 2015) to favor their expansion in the gut lumen. In contrast, $S$. Typhi favors an anti-inflammatory approach by evading (Winter et al., 2015) immune surveillance and colonizing extraintestinal sites such as the gallbladder (Gunn et al., 2014). Reflective of these strategies, different fimbriae are proposed to facilitate nontyphoidal Salmonella's preferential binding to $\mathrm{M}$ cells (Hase et al., 2009) and typhoidal Salmonella's preferential binding to enterocytes (Gonzales et al., 2017). S. Typhimurium FimH, the tip adhesin of Fim, binds glycoprotein 2 (GP2) on M cells in a mannose-dependent manner (Hase et al., 2009), while Lpf facilitates binding to M-like cells (Gonzales et al., 2017) and murine Peyer's patches [Table 1 (Bäumler et al., 1996b)]. Conversely, Stg fimbriae in S. Typhi are thought to promote binding to enterocytes (Gonzales et al., 2017), although in at least some $S$. Typhi the Stg usher protein is likely non-functional due to frame shift mutations leading to pseudogene formation (Townsend et al., 2001; Dufresne et al., 2018).

After breaching the gut epithelium, S. Typhimurium uses Sti, Saf, Agf (curli, non-chaperone-usher fimbriae), and Stc fimbriae at extraintestinal sites, as quadruple deletion mutants show a reduced colonization of the spleen and liver compared to wild type strains (Laniewski et al., 2017). S. Typhi Agf has also been shown to enable binding to the gallbladder epithelium
(Gonzalez-Escobedo and Gunn, 2013), supporting a role for this fimbria in promoting chronic colonization of $S$. Typhi in the gallbladder (Gonzalez-Escobedo et al., 2011). Several fimbriae have been implicated in facilitating long-term intestinal colonization of $129 \mathrm{X} 1 / \mathrm{SvJ}$ [Saf, Bcf, Sti, and Std (Lawley et al., 2006)] and CBA mice [Lpf, Bcf, Stb, Stc, Std, and Sth (Weening et al., 2005)]; as deletion of these fimbriae did not result in a reduced recovery of $S$. Typhimurium from fecal samples of BALB/c mice at five days post infection (Weening et al., 2005), it appears that these fimbriae instead effect colonization in a temporal-manner, at different stages over the course of an infection. Finally, multiple studies have shown that chaperone-usher fimbriae undergo phase variation, a process in which expression of fimbriae in the bacterial population is heterogeneous (Humphries et al., 2001; Humphries et al., 2005; Kolenda et al., 2019). Together, these observations support a role for multiple chaperone-usher fimbriae in facilitating tissue tropism in both acute and chronic infections for both typhoidal and nontyphoidal Salmonella, but also suggest redundant roles for many fimbriae as deletion/inactivation of a single fimbrial gene cluster often has negligible effects on virulence in vivo.

\section{It Is Complicated-A Combination of Fimbrial Adhesin Allelic Diversity, Pseudogene Formation, and Differential Fimbrial Expression Support a Role for Chaperone-Usher Fimbriae in Host Tropism}

Together with the observation that many chaperone-usher fimbriae are distributed across multiple lineages (and serovars), several studies have suggested that the mere presence of fimbrial gene clusters cannot fully explain the observed patterns of host tropism (Chaudhuri et al., 2013; Zhang et al., 2019), highlighting the complexity of the relationship between fimbriae and virulence (Humphries et al., 2001). One possible explanation is that analyses that only consider presence/absence data do not account for pseudogene formation among fimbrial genes, which has been demonstrated previously for multiple host-adapted/ restricted serovars Typhi (Townsend et al., 2001), Dublin (Nuccio and Bäumler, 2014; Langridge et al., 2015), Gallinarum (Clayton et al., 2008), Choleraesuis and Paratyphi C (Nuccio and Bäumler, 2014) (Figure 1C). Another possibility is that the conformation of the tip adhesin mediates binding to different host cells, as several studies have documented that different alleles of fimbrial adhesins are associated with isolation from different hosts (Kisiela et al., 2012; Yue et al.,

TABLE 1 | Summary of fimbriae with known host receptors.

\begin{tabular}{|c|c|c|c|c|}
\hline Chaperone-usher clade & Fimbria & Host cell receptor & Cell types bound ${ }^{1}$ & References \\
\hline$\gamma_{1}$ & Lpf & Unknown & M-like cells & (Bäumler et al., 1996b) \\
\hline$\kappa$ & Pef & Galß1-4(Fuc $\alpha 1-3)$ GlcNAc & Unknown & (Chessa et al., 2008) \\
\hline$\pi$ & Std & $\alpha-1,2$ fucose & Colonic and cecal intestinal epithelial cells & (Chessa et al., 2009; Suwandi et al., 2019) \\
\hline
\end{tabular}

${ }^{1}$ Types of host cells predicted to be the preferential binding target of the fimbria. 
2015; De Masi et al., 2017). Furthermore, there is some evidence to suggest that these adhesins may also use different receptors when infecting different hosts (Grzymajło et al., 2013; Grzymajlo et al., 2017). Several studies have suggested that different conformations of the tip adhesins of Fim (Kisiela et al., 2012; Yue et al., 2015; De Masi et al., 2017), and Bcf and Stf (De Masi et al., 2017) fimbriae among nontyphoidal serovars enhance preferential binding to different host cell lines. For example, the allelic variation in $\mathrm{fimH}$ alleles from host-adapted/hostrestricted serovars (e.g., Dublin and cattle, Typhi and humans, etc.) was associated with preferential binding to cell lines representing the host that the serovar was isolated from (e.g., Dublin and bovine cells) (Yue et al., 2015), corroborating the results of previous studies that suggested that different fim $\mathrm{H}$ alleles allowed for selective binding to different host cell types (Boddicker et al., 2002; Kisiela et al., 2012; Grzymajło et al., 2013). Last, fimbrial gene expression is a complex process that involves regulators encoded in the fimbrial gene cluster that directly regulate transcription of genes in the cluster (Kolenda et al., 2019), as well as global regulators including H-NS (Hurtado-Escobar et al., 2019), CsrA (Sterzenbach et al., 2013), and HdfR (García-Pastor et al., 2019). These complex regulatory pathways may also partially explain why strains representing different serovars show variable expression of fimbriae, and why deletion of these chaperone-usher fimbrial gene clusters in has varying effects on influencing virulence (Azriel et al., 2017).

Overall, these studies suggest that fimbriae-mediated adhesion to host cells is a complex process. Therefore, bigger picture assessments of the roles that these fimbriae play in mediating host interactions will require both an attention to detail (i.e., specific sequence of adhesins, distribution of receptors in different hosts) as well as considerations for the role of phase variation in facilitating evasion of the host immune system (Humphries et al., 2001).

\section{MISSING PIECES OF THE SALMONELLA CHAPERONE-USHER FIMBRIOME PUZZLE}

The appreciable diversity of Salmonella serovars is mirrored by its diverse chaperone-usher fimbriome. While some fimbriae, such as Fim and Agf, have been studied extensively due in part to their expression under certain standard lab culturing conditions (Laniewski et al., 2017; Kolenda et al., 2019), discovery of novel

\section{REFERENCES}

Aviv, G., Elpers, L., Mikhlin, S., Cohen, H., Vitman Zilber, S., Grassl, G. A., et al. (2017). The plasmid-encoded Ipf and Klf fimbriae display different expression and varying roles in the virulence of Salmonella enterica serovar Infantis in mouse vs. avian hosts. PLoS Path. 13, e1006559-e1006559. doi: 10.1371/journal.ppat.1006559

Azriel, S., Goren, A., Shomer, I., Aviv, G., Rahav, G., and Gal-Mor, O. (2017). The Typhi colonization factor (Tcf) is encoded by multiple non-typhoidal Salmonella serovars but exhibits a varying expression profile and fimbrial gene clusters continues with the expanded use of whole genome sequencing in public health and research efforts. With current initiatives aimed at utilizing genomic data for source prediction to facilitate foodborne outbreak investigations (Zhang et al., 2019; Merlotti et al., 2020), an enhanced understanding of the role that diverse chaperone-usher fimbriae, and potentially different conformations of their adhesins, play in the colonization of a wide range of hosts is warranted. Future investigations to understand the sequence diversity and conservation of chaperone-usher fimbriae among lesser studied Salmonella subspecies and serovars will be important for determining patterns of chaperone-usher fimbriae associated with different hosts. Furthermore, structural data of fimbrial components that facilitate interactions with host receptors will require the development and characterization of additional cell culture/organoids and animal models to expand our understanding of the role of chaperone-usher fimbriae in a broader range of tissues and hosts. These, combined with more basic inquiries to understand transcriptional and translational regulatory mechanisms to identify which host signals govern expression of different fimbriae, represent important missing pieces in our understanding of the Salmonella chaperone-usher fimbriome puzzle.

\section{AUTHOR CONTRIBUTIONS}

This review article was conceptualized by RC and MW. RC and MW wrote, reviewed, and edited the original and final drafts. Funding was secured by RC. All authors contributed to the article and approved the submitted version.

\section{FUNDING}

Funding for this review was provided by USDA 2020-6703431905 awarded to RC.

\section{ACKNOWLEDGMENTS}

The authors wish to acknowledge members of the Cornell Food Safety Lab for helpful discussions pertaining to the content of this review.

interchanging contribution to intestinal colonization. Virulence 8, 1791-1807. doi: 10.1080/21505594.2017.1380766

Bäumler, A. J., Tsolis, R. M., Bowe, F. A., Kusters, J. G., Hoffmann, S., and Heffron, F. (1996a). The pef fimbrial operon of Salmonella Typhimurium mediates adhesion to murine small intestine and is necessary for fluid accumulation in the infant mouse. Infect. Immun. 64, 61-68. doi: 10.1128/IAI.64.1.61-68.1996

Bäumler, A. J., Tsolis, R. M., and Heffron, F. (1996b). The lpf fimbrial operon mediates adhesion of Salmonella Typhimurium to murine Peyer's patches. Proc. Natl. Acad. Sci. U. S. A. 93, 279-283. doi: 10.1073/pnas.93.1.279 
Bäumler, A. J., Hargis, B. M., and Tsolis, R. M. (2000). Tracing the origins of Salmonella outbreaks. Science 287, 50-52. doi: 10.1126/science.287.5450.50

Bäumler, A. J., and Heffron, F. (1995). Identification and sequence analysis of I $f A B C D E$, a putative fimbrial operon of Salmonella Typhimurium. J. Bacteriol. 177, 2087-2097. doi: 10.1128/JB.177.8.2087-2097.1995

Boddicker, J. D., Ledeboer, N. A., Jagnow, J., Jones, B. D., and Clegg, S. (2002). Differential binding to and biofilm formation on, HEp-2 cells by Salmonella enterica serovar Typhimurium is dependent upon allelic variation in the fimH gene of the fim gene cluster. Mol. Microbiol. 45, 1255-1265. doi: 10.1046/ j.1365-2958.2002.03121.x

Brenner, F., Villar, R., Angulo, F., Tauxe, R., and Swaminathan, B. (2000). Salmonella nomenclature. J. Clin. Microbiol. 38, 2465-2467. doi: 10.1128/ JCM.38.7.2465-2467.2000

Centers for Disease Control and Prevention (2016). National Enteric Disease Surveillance: Salmonella Annual Report 2016. https://www.cdc.gov/ nationalsurveillance/pdfs/2016-Salmonella-report-508.pdf

Chaudhuri, R. R., Morgan, E., Peters, S. E., Pleasance, S. J., Hudson, D. L., Davies, H. M., et al. (2013). Comprehensive assignment of roles for Salmonella Typhimurium genes in intestinal colonization of food-producing animals. PLoS Genet. 9, e1003456. doi: 10.1371/journal.pgen.1003456

Cheng, R. A., Eade, C. R., and Wiedmann, M. (2019). Embracing diversity: Differences in virulence mechanisms, disease severity, and host adaptations contribute to the success of nontyphoidal Salmonella as a foodborne pathogen. Front. Microbiol. 10, 1368. doi: 10.3389/fmicb.2019.01368

Chessa, D., Dorsey, C. W., Winter, M., and Baümler, A. J. (2008). Binding specificity of Salmonella plasmid-encoded fimbriae assessed by glycomics. J. Biol. Chem. 283, 8118-8124. doi: 10.1074/jbc.M710095200

Chessa, D., Winter, M. G., Jakomin, M., and Bäumler, A. J. (2009). Salmonella enterica serotype Typhimurium Std fimbriae bind terminal $\alpha(1,2)$ fucose residues in the cecal mucosa. Mol. Microbiol. 71, 864-875. doi: 10.1111/j.13652958.2008.06566.x

Clayton, D. J., Bowen, A. J., Hulme, S. D., Buckley, A. M., Deacon, V. L., Thomson, N. R., et al. (2008). Analysis of the role of 13 major fimbrial subunits in colonisation of the chicken intestines by Salmonella enterica serovar Enteritidis reveals a role for a novel locus. BMC Microbiol. 8, 1-15. doi: 10.1186/1471-2180-8-228

Clouthier, S. C., Müller, K. H., Doran, J. L., Collinson, S. K., and Kay, W. W. (1993). Characterization of three fimbrial genes, sefABC, of Salmonella Enteritidis. J. Bacteriol. 175, 2523-2533. doi: 10.1128/JB.175.9.2523-2533.1993

Crump, J. A., Sjölund-Karlsson, M., Gordon, M. A., and Parry, C. M. (2015). Epidemiology, clinical presentation, laboratory diagnosis, antimicrobial resistance, and antimicrobial management of invasive Salmonella infections. Clin. Microbiol. Rev. 28, 901-937. doi: 10.1128/CMR.00002-15

De Masi, L., Yue, M., Hu, C., Rakov, A. V., Rankin, S. C., and Schifferli, D. M. (2017). Cooperation of adhesin alleles in Salmonella-host tropism. mSphere 2, e00066-17. doi: 10.1128/mSphere.00066-17

Desai, P. T., Porwollik, S., Long, F., Cheng, P., Wollam, A., Clifton, S. W., et al. (2013). Evolutionary genomics of Salmonella enterica subspecies. mBio 4, e00579-e00512. doi: 10.1128/mBio.00579-12

Dufresne, K., Saulnier-Bellemare, J., and Daigle, F. (2018). Functional analysis of the chaperone-usher fimbrial gene clusters of Salmonella enterica serovar Typhi. Front. Cell Infect. Microbiol. 8, 26. doi: 10.3389/fcimb.2018.00026

Duguid, J., Anderson, E., and Campbell, I. (1966). Fimbriae and adhesive properties in Salmonellae. J. Path. Bact. 92, 107-137. doi: 10.1002/ path.1700920113

Fàbrega, A., and Vila, J. (2013). Salmonella enterica serovar Typhimurium skills to succeed in the host: virulence and regulation. Clin. Microbiol. Rev. 26, 308-341. doi: 10.1128/CMR.00066-12

Folkesson, A., Advani, A., Sukupolvi, S., Pfeifer, J. D., Normark, S., and Löfdahl, S. (1999). Multiple insertions of fimbrial operons correlate with the evolution of Salmonella serovars responsible for human disease. Mol. Microbiol. 33, 612622. doi: 10.1046/j.1365-2958.1999.01508.x

Fookes, M., Schroeder, G. N., Langridge, G. C., Blondel, C. J., Mammina, C., Connor, T. R., et al. (2011). Salmonella bongori provides insights into the evolution of the Salmonellae. PLoS Pathog. 7, e1002191. doi: 10.1371/ journal.ppat.1002191

García-Pastor, L., Sánchez-Romero, M. A., Jakomin, M., Puerta-Fernández, E., and Casadesús, J. (2019). Regulation of bistability in the std fimbrial operon of
Salmonella enterica by DNA adenine methylation and transcription factors HdfR, StdE and StdF. Nucl. Acid Res. 47, 7929-7941. doi: 10.1093/nar/gkz530

Geibel, S., Procko, E., Hultgren, S. J., Baker, D., and Waksman, G. (2013). Structural and energetic basis of folded-protein transport by the FimD usher. Nature 496, 243-246. doi: 10.1038/nature12007

Gonzales, A. M., Wilde, S., and Roland, K. L. (2017). New insights into the roles of long polar fimbriae and Stg fimbriae in Salmonella interactions with enterocytes and M cells. Infect. Immun. 85, e00172-e00117. doi: 10.1128/ IAI.00172-17

Gonzalez-Escobedo, G., Marshall, J. M., and Gunn, J. S. (2011). Chronic and acute infection of the gall bladder by Salmonella Typhi: understanding the carrier state. Nat. Rev. Microbiol. 9, 9-14. doi: 10.1038/nrmicro2490

Gonzalez-Escobedo, G., and Gunn, J. S. (2013). Gallbladder epithelium as a niche for chronic Salmonella carriage. Infect. Immun. 81, 2920-2930. doi: 10.1128/ IAI.00258-13

Grzymajło, K., Ugorski, M., Kolenda, R., Kędzierska, A., Kuźmińska-Bajor, M., and Wieliczko, A. (2013). FimH adhesin from host unrestricted Salmonella Enteritidis binds to different glycoprotein ligands expressed by enterocytes from sheep, pig and cattle than FimH adhesins from host restricted Salmonella Abortus-ovis, Salmonella Choleraesuis and Salmonella Dublin. Vet. Microbiol. 166, 550-557. doi: 10.1016/j.vetmic.2013.07.004

Grzymajlo, K., Ugorski, M., Suchanski, J., Kedzierska, A. E., Kolenda, R., Jarzab, A., et al. (2017). The novel type 1 fimbriae FimH receptor calreticulin plays a role in Salmonella host specificity. Front. Cell Infect. Microbiol. 7, 326. doi: 10.3389/fcimb.2017.00326

Gunn, J. S., Marshall, J. M., Baker, S., Dongol, S., Charles, R. C., and Ryan, E. T. (2014). Salmonella chronic carriage: epidemiology, diagnosis, and gallbladder persistence. Trends Microbiol. 22, 648-655. doi: 10.1016/j.tim.2014.06.007

Hansmeier, N., Miskiewicz, K., Elpers, L., Liss, V., Hensel, M., and Sterzenbach, T. (2017). Functional expression of the entire adhesiome of Salmonella enterica serotype Typhimurium. Sci. Rep. 7, 1-12. doi: 10.1038/s41598-017-10598-2

Hase, K., Kawano, K., Nochi, T., Pontes, G. S., Fukuda, S., Ebisawa, M., et al. (2009). Uptake through glycoprotein 2 of FimH(+) bacteria by M cells initiates mucosal immune response. Nature 462, 226-230. doi: 10.1038/nature08529

Havelaar, A. H., Kirk, M. D., Torgerson, P. R., Gibb, H. J., Hald, T., Lake, R. J., et al. (2015). World Health Organization global estimates and regional comparisons of the burden of foodborne disease in 2010. PLoS Med. 12, e1001923. doi: 10.1371/journal.pmed.1001923

Hoelzer, K., Moreno Switt, A. I., and Wiedmann, M. (2011). Animal contact as a source of human non-typhoidal salmonellosis. Vet. Res. 42, 34. doi: 10.1186/ 1297-9716-42-34

Humphries, A. D., Townsend, S. M., Kingsley, R. A., Nicholson, T. L., Tsolis, R. M., and Bäumler, A. J. (2001). Role of fimbriae as antigens and intestinal colonization factors of Salmonella serovars. FEMS Microbiol. Lett. 201, 121125. doi: 10.1111/j.1574-6968.2001.tb10744.x

Humphries, A. D., Raffatellu, M., Winter, S., Weening, E. H., Kingsley, R. A., Droleskey, R., et al. (2003). The use of flow cytometry to detect expression of subunits encoded by 11 Salmonella enterica serotype Typhimurium fimbrial operons. Mol. Microbiol. 48, 1357-1376. doi: 10.1046/j.13652958.2003.03507.x

Humphries, A., Deridder, S., and Bäumler, A. J. (2005). Salmonella enterica serotype Typhimurium fimbrial proteins serve as antigens during infection of mice. Infect. Immun. 73, 5329-5338. doi: 10.1128/IAI.73.9.5329-5338.2005

Hurtado-Escobar, G. A., Grépinet, O., Raymond, P., Abed, N., Velge, P., and Virlogeux-Payant, I. (2019). H-NS is the major repressor of Salmonella Typhimurium Pef fimbriae expression. Virulence 10, 849-867. doi: 10.1080/ 21505594.2019.1682752

Issenhuth-Jeanjean, S., Roggentin, P., Mikoleit, M., Guibourdenche, M., De Pinna, E., Nair, S., et al. (2014). Supplement 2008-2010 (no. 48) to the WhiteKauffmann-Le Minor scheme. Res. Microbiol. 165, 526-530. doi: 10.1016/ j.resmic.2014.07.004

Kaper, J. B., Nataro, J. P., and Mobley, H. L. (2004). Pathogenic Escherichia coli. Nat. Rev. Microbiol. 2, 123-140. doi: 10.1038/nrmicro818

Kisiela, D. I., Chattopadhyay, S., Libby, S. J., Karlinsey, J. E., Fang, F. C., Tchesnokova, V., et al. (2012). Evolution of Salmonella enterica virulence via point mutations in the fimbrial adhesin. PLoS Path. 8, e1002733-e1002733. doi: 10.1371/journal.ppat.1002733 
Kolenda, R., Ugorski, M., and Grzymajło, K. (2019). Everything you always wanted to know about Salmonella type 1 fimbriae, but were afraid to ask. Front. Microbiol. 10, 1017. doi: 10.3389/fmicb.2019.01017

Langridge, G. C., Fookes, M., Connor, T. R., Feltwell, T., Feasey, N., Parsons, B. N., et al. (2015). Patterns of genome evolution that have accompanied host adaptation in Salmonella. Proc. Natl. Acad. Sci. 112, 863-868. doi: 10.1073/ pnas. 1416707112

Laniewski, P., Baek, C. H., Roland, K. L., and Curtiss, R.3. (2017). Analysis of spleen-induced fimbria production in recombinant attenuated Salmonella enterica serovar Typhimurium vaccine strains. mBio 8, e01189-e01117. doi: 10.1128/mBio.01189-17

Lawley, T. D., Chan, K., Thompson, L. J., Kim, C. C., Govoni, G. R., and Monack, D. M. (2006). Genome-wide screen for Salmonella genes required for longterm systemic infection of the mouse. PLoS Pathog. 2, e11. doi: 10.1371/ journal.ppat.0020011

Mcclelland, M., Sanderson, K. E., Spieth, J., Clifton, S. W., Latreille, P., Courtney, L., et al. (2001). Complete genome sequence of Salmonella enterica serovar Typhimurium LT2. Nature 413, 852-856. doi: 10.1038/35101614

Merlotti, A., Manfreda, G., Munck, N., Hald, T., Litrup, E., Nielsen, E. M., et al. (2020). Network approach to source attribution of Salmonella enterica serovar Typhimurium and its monophasic variant. Front. Microbiol. 11, 1205. doi: 10.3389/fmicb.2020.01205

Nobbs, A. H., Lamont, R. J., and Jenkinson, H. F. (2009). Streptococcus adherence and colonization. Microbiol. Molec. Biol. Rev. 73, 407-450. doi: 10.1128/ MMBR.00014-09

Nuccio, S. P., and Bäumler, A. J. (2007). Evolution of the chaperone/usher assembly pathway: fimbrial classification goes Greek. Microbiol. Mol. Biol. Rev. 71, 551-575. doi: 10.1128/MMBR.00014-07

Nuccio, S.-P., and Bäumler, A. J. (2014). Comparative analysis of Salmonella genomes identifies a metabolic network for escalating growth in the inflamed gut. $m$ Bio 5, e00929. doi: 10.1128/mBio.00929-14

Paczosa, M. K., and Mecsas, J. (2016). Klebsiella pneumoniae: going on the offense with a strong defense. Microbiol. Molec. Biol. Rev. 80, 629-661. doi: 10.1128/ MMBR.00078-15

Rehman, T., Yin, L., Latif, M. B., Chen, J., Wang, K., Geng, Y., et al. (2019). Adhesive mechanism of different Salmonella fimbrial adhesins. Microb. Path. 137, 103748. doi: 10.1016/j.micpath.2019.103748

Rivera-Chavez, F., and Bäumler, A. J. (2015). The pyromaniac inside you: Salmonella metabolism in the host gut. Annu. Rev. Microbiol. 69, 31-48. doi: 10.1146/annurev-micro-091014-104108

Sterzenbach, T., Nguyen, K. T., Nuccio, S. P., Winter, M. G., Vakulskas, C. A., Clegg, S., et al. (2013). A novel CsrA titration mechanism regulates fimbrial gene expression in Salmonella Typhimurium. EMBO J. 32, 2872-2883. doi: 10.1038/emboj.2013.206

Stubenrauch, C. J., Dougan, G., Lithgow, T., and Heinz, E. (2017). Constraints on lateral gene transfer in promoting fimbrial usher protein diversity and function. Open Biol. 7, 170144. doi: 10.1098/rsob.170144

Suwandi, A., Galeev, A., Riedel, R., Sharma, S., Seeger, K., Sterzenbach, T., et al. (2019). Std fimbriae-fucose interaction increases Salmonella-induced intestinal inflammation and prolongs colonization. PLoS Path. 15, e1007915. doi: 10.1371/journal.ppat.1007915

Thanassi, D. G., Nuccio, S. P., Shu, S. K. S., and Bäumler, A. (2007). Fimbriae: Classification and Biochemistry. EcoSal. Plus 2. doi: 10.1128/ecosal.2.4.2.1

Thomson, N. R., Clayton, D. J., Windhorst, D., Vernikos, G., Davidson, S., Churcher, C., et al. (2008). Comparative genome analysis of Salmonella Enteritidis PT4 and Salmonella Gallinarum 287/91 provides insights into evolutionary and host adaptation pathways. Genome Res. 18, 1624-1637. doi: 10.1101/gr.077404.108
Torres, A. G., Giron, J. A., Perna, N. T., Burland, V., Blattner, F. R., Avelino-Flores, F., et al. (2002). Identification and Characterization of $l p f A B C C^{\prime} D E$, a fimbrial operon of Enterohemorrhagic Escherichia coli O157:H7. Infect. Immun. 70, 5416-5427. doi: 10.1128/IAI.70.10.5416-5427.2002

Townsend, S. M., Kramer, N. E., Edwards, R., Baker, S., Hamlin, N., Simmonds, M., et al. (2001). Salmonella enterica serovar Typhi possesses a unique repertoire of fimbrial gene sequences. Infect. Immun. 69, 2894-2901. doi: 10.1128/IAI.69.5.2894-2901.2001

Tsolis, R. M., Townsend, S. M., Miao, E. A., Miller, S. I., Ficht, T. A., Adams, L. G., et al. (1999). Identification of a putative Salmonella enterica serotype Typhimurium host range factor with homology to IpaH and YopM by signature-tagged mutagenesis. Infect. Immun. 67, 6385-6393. doi: 10.1128/ IAI.67.12.6385-6393.1999

Tsolis, R. M., and Bäumler, A. J. (2020). Gastrointestinal host-pathogen interaction in the age of microbiome research. Curr. Opin. Microbiol. 53, 78-89. doi: 10.1016/j.mib.2020.03.002

Wagner, C., and Hensel, M. (2011). "Adhesive mechanisms of Salmonella enterica," in Bacterial adhesion Eds. A. Goldman (Heidelberg, Germany: Springer), vol. 17-34. doi: 10.1007/978-94-007-0940-9_2

Weening, E. H., Barker, J. D., Laarakker, M. C., Humphries, A. D., Tsolis, R. M., and Bäumler, A. J. (2005). The Salmonella enterica serotype Typhimurium lpf, $\mathrm{bcf}, \mathrm{stb}$, stc, std, and sth fimbrial operons are required for intestinal persistence in mice. Infect. Immun. 73, 3358-3366. doi: 10.1128/IAI.73.6.3358-3366.2005

Wilksch, J. J., Yang, J., Clements, A., Gabbe, J. L., Short, K. R., Cao, H., et al. (2011). $\mathrm{MrkH}$, a novel c-di-GMP-dependent transcriptional activator, controls Klebsiella pneumoniae biofilm formation by regulating type 3 fimbriae expression. PLoS Pathog. 7, e1002204. doi: 10.1371/journal.ppat.1002204

Winter, S. E., Winter, M. G., Atluri, V., Poon, V., Romão, E. L., Tsolis, R. M., et al. (2015). The flagellar regulator TviA reduces pyroptosis by Salmonella enterica serovar Typhi. Infect. Immun. 83, 1546-1555. doi: 10.1128/IAI.02803-14

Worley, J., Meng, J., Allard, M. W., Brown, E. W., and Timme, R. E. (2018). Salmonella enterica phylogeny based on whole-genome sequencing reveals two new clades and novel patterns of horizontally acquired genetic elements. mBio 9, e02303-e02318. doi: 10.1128/mBio.02303-18

Wurpel, D. J., Beatson, S. A., Totsika, M., Petty, N. K., and Schembri, M. A. (2013). Chaperone-usher fimbriae of Escherichia coli. PLoS One 8, e52835-e52835. doi: 10.1371/journal.pone.0052835

Yue, M., Rankin, S. C., Blanchet, R. T., Nulton, J. D., Edwards, R. A., and Schifferli, D. M. (2012). Diversification of the Salmonella fimbriae: a model of macro- and microevolution. PLoS One 7, e38596-e38596. doi: 10.1371/journal.pone.0038596

Yue, M., Han, X., De Masi, L., Zhu, C., Ma, X., Zhang, J., et al. (2015). Allelic variation contributes to bacterial host specificity. Nat. Commun. 6, 8754. doi: 10.1038/ncomms 9754

Zhang, S., Li, S., Gu, W., Den Bakker, H., Boxrud, D., Taylor, A., et al. (2019). Zoonotic source attribution of Salmonella enterica serotype Typhimurium using genomic surveillance data, United States. Emerg. Infect. Dis. 25, 82-91. doi: $10.3201 /$ eid2501.180835

Conflict of Interest: The authors declare that the research was conducted in the absence of any commercial or financial relationships that could be construed as a potential conflict of interest.

Copyright $\odot 2021$ Cheng and Wiedmann. This is an open-access article distributed under the terms of the Creative Commons Attribution License (CC BY). The use, distribution or reproduction in other forums is permitted, provided the original author(s) and the copyright owner(s) are credited and that the original publication in this journal is cited, in accordance with accepted academic practice. No use, distribution or reproduction is permitted which does not comply with these terms. 